\title{
Análise do inventário de consciência metacognitiva de alunos do curso técnico em enfermagem
}

\author{
Analysis of the metacognitive awareness inventory of students in the nursing technical course \\ Análisis del inventario de conciencia metacognitiva de estudiantes del curso técnico de enfermería
}

Recebido: 30/08/2021 | Revisado: 07/09/2021 | Aceito: 11/09/2021 | Publicado: 14/09/2021

Luciana Rocha dos Santos

ORCID: https://orcid.org/0000-0002-4714-9801

Universidade Federal do Rio de Janeiro, Brasil

E-mail: lurochas@yahoo.com.br

Mauricio Abreu Pinto Peixoto

ORCID: https://orcid.org/0000-0002-2604-279X

Universidade Federal do Rio de Janeiro, Brasil

E-mail: geac.ufrj@gmail.com

\begin{abstract}
Resumo
Este estudo teve como objetivo obter um diagnóstico da percepção metacognitiva dos educandos de um curso técnico em enfermagem, e apontar caminhos para uma aprendizagem autônoma. Para tanto, realizou-se um survey interseccional com 65 estudantes de um curso técnico em enfermagem, durante o ensino remoto emergencial. O Metacognitive Awareness Inventory (MAI) foi utilizado para investigar a disposição do aluno em relação ao seu conhecimento metacognitivo e regulação cognitiva, o que implica na avaliação da capacidade para refletir e na tomada de decisão. Observou-se 66\% de Conhecimento metacognitivo e 68\% de Regulação da Cognição. O primeiro fator compôs-se de Conhecimentos condicional (69\%), Declarativo (66\%) e Processual (62\%). Já a Regulação da Cognição distribuiu-se entre Planejamento (68\%), Gestão da Informação (69\%), Monitoramento (67\%), Depuração (82\%) e Avaliação (63\%). Tais resultados, entre outros, apontaram para a necessidade de aprimoramento e ampliação da consciência metacognitiva, através da inserção de estratégias de estímulo à cultura do pensar, a reflexão crítica, a autorregulação da aprendizagem e a aprender a aprender.
\end{abstract}

Palavras-chave: Metacognição; MAI; Técnico em Enfermagem.

\begin{abstract}
This study aimed to obtain a diagnosis of the metacognitive perception of students from a technical nursing course, and to point out paths for autonomous learning. For that, an intersectional survey was carried out with 65 students of a technical course in nursing, during emergency remote teaching. The Metacognitive Awareness Inventory (MAI) was used to investigate the student's disposition in relation to their metacognitive knowledge and cognitive regulation, which implies the assessment of the ability to reflect and decision-making. $66 \%$ of metacognitive knowledge and $68 \%$ of regulation of cognition were observed. The first factor was composed of Conditional Knowledge (69\%), Declarative (66\%) and Procedural (62\%). On the other hand, Cognition Regulation was distributed among Planning (68\%), Information Management (69\%), Monitoring (67\%), Debugging (82\%) and Evaluation (63\%). Such results, among others, pointed to the need for improvement and expansion of metacognitive awareness, through the insertion of strategies to stimulate the culture of thinking, critical reflection, self-regulation of learning and learning to learn.
\end{abstract}

Keywords: Metacognition; MAI; Nursing Technical.

\begin{abstract}
Resumen
Este estudio tuvo como objetivo obtener un diagnóstico de la percepción metacognitiva de los estudiantes de un curso técnico de enfermería y señalar caminos para el aprendizaje autónomo. Por lo tanto, se realizó una encuesta interseccional con 65 estudiantes de un curso técnico de enfermería durante la enseñanza remota de emergencia. Se utilizó el Inventario de Conciencia Metacognitiva (MAI) para investigar la disposición del estudiante en relación a su conocimiento metacognitivo y regulación cognitiva, lo que implica la evaluación de la capacidad de reflexión y toma de decisiones. Se observó el 66\% del conocimiento metacognitivo y el 68\% de la regulación de la cognición. El primer factor estuvo compuesto por Conocimiento Condicional (69\%), Declarativo (66\%) y Procesal (62\%). Por otro lado, la Regulación cognitiva se distribuyó entre Planificación (68\%), Gestión de la información (69\%), Monitoreo (67\%), Depuración (82\%) y Evaluación (63\%). Dichos resultados, entre otros, señalaron la necesidad de mejorar y ampliar la conciencia metacognitiva, a través de la inserción de estrategias para estimular la cultura del pensamiento, la reflexión crítica, la autorregulación del aprendizaje y el aprender a aprender.
\end{abstract}

Palabras clave: Metacognición; MAI; Técnico de Enfermería. 


\section{Introdução}

O profissional técnico em enfermagem realiza atividades de assistência, prevenção, controle e execução da prestação de cuidados diretos aos pacientes, assistindo ao enfermeiro, em empresas públicas e privadas, como hospitais e clínicas. Dada a sua importância na prestação do cuidado e assistência à saúde, são demandados conhecimentos que vão além de suas motivações e competências técnicas.

Nesse sentido, podemos ter como motivações profissionais do técnico em enfermagem, o sentimento de realização e responsabilidade profissional, além do incentivo externo como, por exemplo, expectativa em prosseguir nos estudos, visando a graduação na área da saúde (medicina, odontologia, enfermagem), mais frequente em alunos da modalidade de ensino médio técnico integrado; e, inserção no mercado de trabalho em curto prazo, almejado por alunos da modalidade de ensino técnico subsequente, para o suprimento das necessidades da vida em sociedade e a possibilidade de mobilidade social (Marin et al., 2014; Wermelinger, 2007).

Quanto às competências esperadas por gestores de enfermagem para os técnicos em enfermagem, pressupõe-se capacidades de agir, mobilizar e transferir conhecimentos na solução de situações que favoreçam a prestação de uma assistência integral e humanizada ao paciente (Silva et al., 2018). No entanto, o profissional técnico em enfermagem é preparado para realizar procedimentos técnicos e não recebe uma formação integrada, crítica e reflexiva (Serradilha, Duarte, \& Tonete, 2019).

A realidade emergente com a pandemia da COVID-19 demandou formas alternativas de ensino e expôs diversas dificuldades da educação no Brasil, como o acesso à internet de qualidade e preparo das instituições públicas. Em um primeiro momento, foi adotado o ensino remoto emergencial (ERE), em que as disciplinas foram transpostas para meios alternativos, como TV e internet (Brasil, 2020; Hodges et al., 2020). Porém, com a necessidade de manutenção do distanciamento social, a adoção do ensino híbrido, modalidade que mescla parte do ensino presencial com parte virtual (Bacich, Tanzi Neto, \& Trevisani, 2015), passou a ser empregada por boa parte das instituições, constituindo mais um desafio para a formação desses profissionais.

Nessas modalidades de ensino-aprendizagem há necessidade de maior autonomia dos alunos, tomada de consciência e capacidade de reflexão, corroborando com competências esperadas para estes futuros profissionais. Para tanto, conhecer a capacidade metacognitiva, como o conhecimento metacognitivo e a regulação cognitiva, podem auxiliar o aluno a direcionar o esforço necessário para compreender o contexto, tendo em vista adequar ações necessárias e gerenciar o próprio aprendizado, habilidades indispensáveis para um profissional da área de saúde reflexivo e autônomo.

O entendimento que as pessoas possuem sobre o próprio processamento cognitivo é designado pela Psicologia Cognitiva como metacognição. A metacognição, para Flavell, Miller e Miller (1999, p. 125) é definida como a "cognição acerca da cognição". Peixoto, Brandão e Santos (2007, p. 69), esclarecem que “a metacognição é um termo usado para descrever diferentes aspectos do conhecimento que construímos sobre como nós percebemos, recordamos, pensamos e agimos".

John Hurley Flavell, psicólogo norte americano especialista em psicologia cognitiva e um dos pioneiros no estudo da metacognição, sugere que a melhoria do conhecimento metacognitivo e habilidades de monitoramento cognitivo, podem influenciar positivamente nos resultados da aprendizagem (Flavell, 1979). Esse autor descreveu a metacognição como constituída por quatro componentes: conhecimento metacognitivo, experiências metacognitivas, objetivos ou tarefas, e ações ou estratégias. O conhecimento metacognitivo é o conhecimento armazenado pela pessoa de si com relação às diversas tarefas, objetivos, ações e experiências cognitivas. Este conhecimento se relaciona às crenças que a pessoa possui de suas habilidades ou deficiências. Já as experiências metacognitivas são quaisquer experiências cognitivas ou afetivas conscientes, como por exemplo a sensação ou sentimento de compreensão ou não de dado conteúdo. A interação entre conhecimento metacognitivo e 
experiências metacognitivas interferem um no outro, na medida em que ocorre o monitoramento contínuo e a regulação de conhecimentos na escolha e implementação adequada das estratégias de aprendizagem (Santos et al., 2021).

O processo metacognitivo, está ligado a capacidade do ser humano de ter consciência de seus atos e pensamentos, de monitorar e autorregular; ou seja, é o processo em que o indivíduo ao realizar ações cognitivas, acompanha as ações enquanto acontecem, correspondendo a capacidade de reflexão consciente sobre estas ações (Jou \& Sperb, 2006).

Kuhn (2000) defende que aumentar a consciência e o controle metacognitivo, possibilita desenvolvimento mental, reconhecimento de objetivos, seleção e uso de estratégias, interpretação de evidências e monitoramento ativo, aprimorando o desenvolvimento metacognitivo. Como destaca Pintrich (2002), os estudantes que desenvolvem a consciência e o conhecimento metacognitivo, se tornam mais conscientes de seus próprios pensamentos, mais conhecedores da cognição em geral e tendem a aprender melhor. Além disso, Rosa et al. (2020) salientam que o contexto pode acionar a conscientização e incitar um processo reflexivo metacognitivo, por exemplo, através de atividades pedagógicas direcionadas.

Deste modo, um componente chave para a metacognição é a consciência dos próprios pensamentos e reflexão nos processos de aprendizagem. Portanto, entender como os alunos pensam e controlam seu processo cognitivo, faz-se necessário para a proposição de intervenções acertadas em direção a um aluno metacognitivo e um futuro profissional mais eficiente (Ertmer \& Newby, 1996; Xavier, Peixoto, \& Veiga, 2021).

Diante disso, foi realizado o levantamento da consciência metacognitiva dos alunos do curso técnico em enfermagem, por meio do inventário de consciência metacognitiva (Metacognitive Awareness Inventory - MAI), proposto por Schraw e Dennison (1994), para investigar a disposição do aluno em relação ao conhecimento metacognitivo e regulação cognitiva, e apontar caminhos no sentido de promover um aprendiz reflexivo e autônomo, capaz de gerenciar seu próprio aprendizado.

\section{Metodologia}

Survey interseccional (Babbie, 2003), que buscou analisar a percepção dos alunos, no que concerne à sua própria aprendizagem, à luz dos conceitos de metacognição apresentados por Flavell (1979), utilizando um questionário de auto relato, o inventário de consciência metacognitiva, proposto por Schraw e Dennison (1994).

Os participantes da pesquisa foram alunos matriculados no curso técnico em enfermagem, de uma escola técnica pública do Estado do Rio de Janeiro, cuja participação ocorreu de forma espontânea e voluntária. O questionário foi implementado em um formulário Google, com respostas anônimas. Após a pesquisa os dados foram compilados e analisados com a finalidade de entender o pensamento metacognitivo do aluno frente ao contexto, e refletir sobre caminhos que possam promover a aprendizagem consciente, reflexiva e autônoma.

Considerando tratar-se de uma pesquisa de dados anônimos e apresentação de resultados de forma compilada, não foi solicitado parecer do Comitê de Ética em Pesquisa, conforme determina o parágrafo único, inciso I, do artigo $1^{\circ}$, da Resolução 510/2016 do Conselho Nacional de Saúde (Brasil, 2016).

\subsection{0 instrumento de coleta}

O Inventário de Consciência Metacognitiva, proposto por Schraw e Dennison (1994) é um questionário metacognitivo de fácil realização, adequado para mensurar a consciência metacognitiva de adolescentes e adultos, composto por 52 questões (Quadro 1). 
Quadro 1: Questões do inventário da consciência metacognitiva.

\begin{tabular}{|l|}
\multicolumn{1}{|c|}{ Itens do Inventário de Consciência Metacognitiva (MAI) } \\
\hline 1. Quando estudo, frequentemente estabeleço objetivos a serem alcançados. \\
\hline 2. Considero várias alternativas para um problema antes de respondê-lo. \\
\hline 3. Tento usar estratégias que deram certo no passado. \\
\hline 4. Enquanto aprendo, procuro estabelecer um ritmo apropriado para o tempo que disponho. \\
\hline 5. Tenho consciência dos meus talentos e limitações intelectuais. \\
\hline 6. Penso sobre o que realmente preciso saber antes de começar uma tarefa. \\
\hline 7. Quando termino de fazer um teste, geralmente, sei como me saí nele. \\
\hline 8. Costumo definir metas específicas antes de começar uma tarefa. \\
\hline 9. Procuro prestar mais atenção quando me deparo com informações importantes. \\
\hline 10. Sei que tipo de informação é mais importante para aprender. \\
\hline 11. Antes de finalizar a resolução de um problema, pergunto-me se considerei todas as diferentes possibilidades de \\
resolução.
\end{tabular}
resolução.

12. Sou bom em organizar informações.

13. Conscientemente foco atenção em informações importantes.

14.Tenho propósitos específicos para cada estratégia que uso.

15. Aprendo melhor quando já sei alguma coisa sobre o assunto.

16. Sei o que o professor espera que eu aprenda.

17. Sou bom em lembrar informações.

18. Uso diferentes estratégias de aprendizagem, dependendo da situação.

19. Pergunto-me se haveria uma maneira mais fácil de fazer a coisa depois que eu termino uma tarefa.

20. Tenho controle sobre o quanto estou aprendendo.

21. Costumo rever pontos que me ajudem a entender as relações importantes.

22. Procuro elaborar perguntas sobre o material antes de começar a estudar.

23. Penso em várias maneiras de resolver um problema e tento escolher a melhor.

24. Posso resumir o que aprendi depois que termino de estudar.

25. Peço ajuda a outros quando não entendo alguma coisa.

26. Quando preciso, sou capaz de me motivar para aprender.

27. Estou ciente das estratégias de aprendizagem que uso quando estou estudando.

28. Procuro analisar a utilidade das estratégias enquanto estudo.

29. Uso os meus talentos intelectuais para compensar minhas limitações.

30. Concentro-me sobre o significado e a importância de novas informações.

31. Crio meus próprios exemplos para tornar a informação mais significativa.

32. Sou capaz de avaliar bem o quanto entendo de alguma coisa.

33. Encontro e uso estratégias de aprendizagem úteis automaticamente.

34. Costumo parar regularmente para verificar minha compreensão.

35. Sei o quanto cada estratégia que uso será mais eficaz.

36. Quando finalizo uma tarefa, me pergunto o quão bem cumpri meus objetivos.

37. Costumo usar imagens e diagramas para me ajudar a entender e aprender.

38. Pergunto-me se considerei todas as opções após resolver um problema.

39. Tento traduzir novas informações em minhas próprias palavras.

40. Mudo de estratégia quando não consigo entender.

41. Tento usar a estrutura organizacional do texto para me ajudar a compreendê-lo.

42. Leio cuidadosamente as instruções antes de começar uma tarefa.

43. Pergunto-me se o que estou lendo tem relação com o que eu já sei.

44. Tento reavaliar minhas suposições quando fico confuso.

45. Tento organizar meu tempo para cumprir melhor meus objetivos.

46. Aprendo mais quando estou interessado no tema.

47. Tento dividir o que tenho para estudar em etapas menores.

48. Concentro-me no sentido global ao invés dos detalhes.

49. Frequentemente me pergunto sobre como está meu desempenho enquanto estou aprendendo algo novo.

50. Assim que finalizo uma tarefa, pergunto-me se eu aprendi tanto quanto eu podia.

51. Paro e volto quando encontro uma nova informação que não ficou clara.

52. Paro e releio quando fico confuso.

Fonte: Adaptado de Schraw e Dennison (1994) e Lima Filho e Bruni (2015). 
O instrumento que abrange dois aspectos importantes da metacognição, o conhecimento metacognitivo e a regulação da cognição, sendo que cada um destes aspectos contempla subprocessos, como disposto no Quadro 2 (Schraw \& Dennison, 1994; Peixoto, Brandão \& Tavares, 2021).

Quadro 2: Categorias, Fatores Metacognitivos e questões correspondentes do MAI.

\begin{tabular}{|c|l|l|}
\hline $\begin{array}{c}\text { Categorias } \\
\text { Metacognitivas }\end{array}$ & \multicolumn{1}{|c|}{ Fatores Metacognitivos } & \multicolumn{1}{c|}{ Questões do MAI } \\
\hline \multirow{2}{*}{$\begin{array}{c}\text { 1. Conhecimento } \\
\text { metacognitivo }\end{array}$} & 1.1 Conhecimento Declarativo & $5,10,12,16,17,20,32$ e 46 \\
\cline { 2 - 3 } & 1.2 Conhecimento Processual & $3,14,27$ e 33 \\
\cline { 2 - 3 } & 1.3 Conhecimento Condicional & $15,18,26,29$ e 35 \\
\hline \multirow{4}{*}{$\begin{array}{c}\text { 2. Regulação da } \\
\text { cognição }\end{array}$} & 2.1 Planejamento & $4,6,8,22,23,42$ e 45 \\
\cline { 2 - 3 } & 2.2 Gestão da Informação & $9,13,30,31,37,39,41,43,47$ e 48 \\
\cline { 2 - 3 } & 2.3 Monitoramento & $1,2,11,21,28,34$ e 49 \\
\cline { 2 - 3 } & 2.4 Depuração & $25,40,44,51$ e 52 \\
\hline
\end{tabular}

Fonte: Adaptado de Schraw e Dennison (1994) e Lima Filho e Bruni (2015).

Em relação ao conhecimento sobre cognição, Schraw e Dennison (1994) definiram questões para três subprocessos, denominados: conhecimento declarativo, que representam o conhecimento sobre si mesmo e sobre estratégias; conhecimento processual, ou conhecimento sobre como usar estratégias; e, o conhecimento condicional, que reflete o conhecimento sobre quando e por que usar estratégias.

Quanto a regulação da cognição, as questões abrangem cinco subprocessos que refletem o controle da aprendizagem: planejamento, que são a definição de metas e alocação de recursos; estratégias de gerenciamento de informações, que correspondem a implementação de estratégias e heurísticas; monitoramento de compreensão, responsável pela auto avaliação da aprendizagem; estratégias de depuração, que ocorrem para corrigir erros de desempenho; e avaliação, que constituem a análise efetiva do desempenho (Schraw \& Dennison, 1994; Lima Filho, 2013; Lima Filho \& Bruni, 2015; Peixoto, Brandão \& Tavares, 2021).

\subsection{Contexto da pesquisa}

A pesquisa da consciência metacognitiva foi proposta para todos os alunos do curso técnico em enfermagem, de uma escola técnica estadual da rede FAETEC (Fundação de Apoio à Escola Técnica do Estado do Rio de Janeiro), incluindo as modalidades, ensino médio técnico integrado e técnico subsequente.

Devido a pandemia da COVID-19, a coleta ocorreu durante o período de ensino remoto emergencial. Nesse período, as aulas regulares presenciais foram suspensas e os conteúdos disponibilizados no ambiente virtual de aprendizagem Moodle (Modular Object-Oriented Dynamic Learning Environment) e em um blog. Vários alunos declararam não ter acesso à internet ou ter acesso precário, dificultando o acompanhamento das atividades de ensino. As atividades remotas foram organizadas pelos professores em trilhas de aprendizagem (Santos et al., 2021), indicando materiais, vídeos, áudios e tarefas de fixação.

O convite para participação na pesquisa foi feito coletivamente por meio de mensagens postadas nos grupos de WhatsApp de cada turma, que incluía link para o questionário. Os alunos que aceitaram participar responderam de forma anônima e online ao questionário criado no Google Forms. Foram realizados quatro reenvios, buscando alcançar o maior número de alunos possível, perfazendo 26 dias de coleta, no período de 14 de junho a 10 de julho de 2020.

O questionário englobou três partes: 1) questões gerais como turma, idade e sexo; 2) MAI - 52 itens, de resposta fechada e obrigatórias utilizando uma escala tipo Likert de três pontos, constando: sempre, às vezes e nunca; e, 3) três questões 
abertas e não obrigatórias: 1. Você gostaria de complementar alguma questão? 2. O que você percebe de diferença, em relação a sua aprendizagem, no ensino presencial e no ensino remoto? 3. Você faria aulas online para aprender a estudar melhor? Visando conhecer dificuldades enfrentadas pelo aluno, sua vivência e pensamento, e encontrar pistas para a melhoria de seu aprendizado.

A escala Likert foi reduzida para três opções devido ao número elevado de questões, da pouca experiência dos alunos com o tema pesquisado, dificuldades para o esclarecimento de dúvidas online, e ainda, visando baixo consumo de internet, já que parcela importante dos sujeitos não tinha condições ideais de acesso à rede.

Os resultados foram coletados em planilha Google e categorizados conforme o Quadro 2, que apresenta as categorias conhecimento metacognitivo (CM) e regulação cognitiva (RC), seus respectivos fatores e questões do MAI correspondentes (Quadro 1).

\section{Resultados}

Participaram da pesquisa, de forma voluntária, um total de 65 estudantes dos 171 matriculados no curso técnico em enfermagem, em 2020, equivalente a 38\% do total, e englobou todas as séries do diurno e noturno da escola. Majoritariamente, obteve-se $88 \%$ dos respondentes do sexo feminino e $12 \%$ do sexo masculino, com idades entre 15 e 43 anos, sendo $54 \%$ com idade inferior a 18 anos.

O MAI reúne perguntas sobre como um indivíduo pensa e age diante de questões relativas ao seu aprendizado. Estas perguntas visam identificar o pensamento e atitudes classificadas segundo duas categorias: Conhecimento Metacognitivo (CM) e Regulação da Cognição (RC). Sendo estas subdivididas em três fatores, no caso do CM, e cinco fatores para a RC (Quadro 2). Os resultados foram apresentados em termos de percentuais de cada fator em relação ao total de cada categoria (CM e RC).

Para apurar estes resultados, primeiramente cada resposta recebeu pontuação zero (0), cinco (5) e dez (10), respectivamente, para resultados nunca, às vezes e sempre. Em seguida, a pontuação de cada fator foi obtida pela soma de todos os valores equivalentes às questões conforme o Quadro 2. A soma dos fatores de cada categoria resultou nos valores do CM e da RC. A porcentagem de cada fator foi obtida após o cálculo da pontuação de cada fator em relação ao valor correspondente ao total para cada categoria. O cálculo da porcentagem média por categoria resultou da soma dos valores percentuais dos respectivos fatores de cada sujeito e divididos pelo total de sujeitos (65).

A pesquisa apontou que a média geral, que englobou todos os participantes, os resultados foram: $66 \%$ de conhecimento metacognitivo e $68 \%$ de regulação da cognição. Partindo destes percentuais percebe-se que, em geral, os alunos apresentaram um resultado razoável, tanto para o conhecimento de seu próprio conhecimento (CM), quanto para a habilidade de monitoramento e controle de seu conhecimento (RC).

O resultado do cômputo por fatores mostra certa constância e proximidade com os valores percentuais médios, tanto para o CM quanto para a RC, na maioria dos fatores. A exceção está no fator depuração, cujo valor foi de $82 \%$ (Figura 1).

Em relação aos fatores relativos ao conhecimento metacognitivo, o maior percentual ocorreu para o fator conhecimento condicional (69\%), seguido pelo conhecimento declarativo (66\%) e após conhecimento processual (62\%) (Figura 1). Os percentuais referentes ao conhecimento metacognitivo, sugerem que os alunos têm maior percepção de quando e porque utilizar as estratégias de estudo que conhecem (conhecimento condicional de 69\%), porém, menor percepção de como usar essas estratégias, visto que o fator conhecimento processual foi de $62 \%$. Quanto aos fatores relativos à regulação da cognição, o maior percentual foi $82 \%$ para depuração, que representa alta habilidade em corrigir erros de desempenho, e o menor percentual foi 63\% para o fator avaliação e análise de desempenho de aprendizagem (Figura 1). Também é importante salientar que estes são percentuais médios do grupo pesquisado. Contudo, os resultados individuais apontaram importante variação, ocorrendo entre $25 \%$ e $100 \%$ em alguns fatores. 
Figura 1: Gráfico do percentual dos fatores: conhecimento metacognitivo $(\mathrm{CM})$ e regulação da cognição (RC).

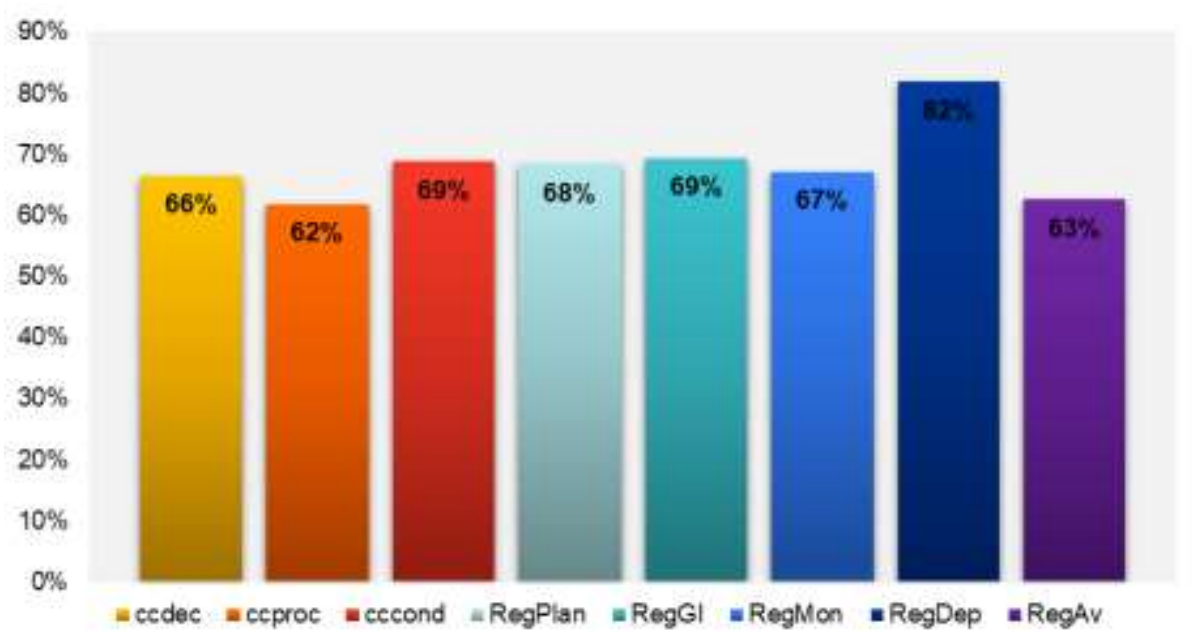

Legenda: conhecimento declarativo (ccdec), conhecimento processual (ccproc), conhecimento condicional (cccond), planejamento (RegPlan), gerenciamento de informações (RegGI), monitoramento (RegMon), depuração (RegDep) e avaliação (RegAv). Fonte: Autores.

Do ponto de vista individual, a pesquisa apontou que apenas cinco (05) alunos demonstraram alta consciência metacognitiva, com percentual acima de $81 \%$, tanto para o CM quanto para a RC, assim como revelou dois (02) alunos com percentuais abaixo de $38 \%$ em ambas categorias.

Também foi possível observar, no resultado individual, que alguns alunos possuem percepção da consciência metacognitiva inversamente proporcional, com baixo percentual de conhecimento metacognitivo (35\%) e alta regulação cognitiva (66\%); ou, ao contrário, alto percentual de conhecimento metacognitivo (85\%) e baixa regulação cognitiva (69\%). Ou seja, alguns alunos demonstraram que conhecem pouco de si, de estratégias e como usá-las, mas possuem uma percepção de que sabem controlar e monitorar seu aprendizado e vice-versa.

Ao analisar os dados por turma, idade e sexo, não se constatou diferença significativa, pois os resultados pouco variaram em torno da média. Assim, em relação ao CM, o menor valor percentual foi de $64 \%$ para alunos maiores de 18 anos e do $3^{\circ}$ ano diurno, e maior valor para alunos do sexo masculino, com $71 \%$. Em relação a RC, o menor valor foi de $65 \%$, para a turma do $3^{\circ}$ módulo noturno e o maior valor foi de $71 \%$, para a turma do $1^{\circ}$ ano diurno e do $1^{\circ}$ módulo noturno.

Cabe salientar que a maioria das perguntas do MAI receberam a resposta "Às vezes" (48\%), seguido de "Sempre" (44\%) e "Nunca" (8\%). Olhando para todos os fatores, apenas Planejamento e Depuração tiveram maioria de respostas "Sempre" para seus itens, isto é, os alunos identificam de imediato maior certeza ou confiança ao marcar determinada resposta nestes fatores. Os demais fatores e seus respectivos itens do questionário receberam maioria de "Às vezes", o que pode significar que nem sempre ou de vez em quando, ou até poucas vezes, os alunos identificaram que realizavam estes itens, que correspondem aos fatores: conhecimento declarativo, conhecimento processual, conhecimento condicional, gestão da informação, monitoramento e avaliação.

Em relação às perguntas discursivas, na questão 1, sobre o desejo de comentar alguma questão, a grande maioria optou por não fazê-lo, sendo que um aluno escreveu não ter entendido a maioria das questões e outro afirmou sempre usar a mesma estratégia (“A maioria delas não entendi!” e "Questão 40. Eu sempre uso a mesma estratégia em todas as matérias...”).

A questão 2, sobre a diferença entre aulas presenciais e remotas, a maioria relatou que o presencial é mais fácil, pela explicação do professor, oportunidade de tirar dúvidas e interação com os colegas (“No presencial o professor tira nossas dúvidas e a interação com os colegas ajuda a repensar"), pois no remoto ocorre, por exemplo, distração e perda de foco ("No remoto é como andar no escuro, pouco foco, muita interrupção, pouco aprendizado e sem ânimo de correr atrás”). Entretanto, um estudante afirmou não ter qualquer dificuldade, afirmando inclusive que "No presencial é mais complicado do que online". 
Na questão 3, sobre o interesse em realizar aulas que pudesse ajudar a aprender a aprender, a resposta foi positiva, $57 \%$ dos alunos responderam sim ao questionamento, demonstrando estarem receptivos a conhecer orientações que os auxiliem a melhorar seus resultados de aprendizagem.

\section{Discussão}

Schraw e Dennison (1994) realçam que a metacognição diz respeito à capacidade de reconhecer, refletir, entender e controlar o aprendizado, e propõem captar estas capacidades sob dois aspectos, o conhecimento metacognitivo e a regulação cognitiva. O conhecimento metacognitivo inclui conhecimento tanto da pessoa, objetivos e estratégias, quanto conhecimento das condições sob as quais essas estratégias podem ser usadas, do grau em que as estratégias são eficazes e de experiências cognitivas com as estratégias (Pintrich, 2002; Peixoto, Brandão \& Tavares, 2021; Santos et al., 2021). A percepção desse conhecimento (conhecimento do conhecimento) contribui para a realização da regulação cognitiva consciente, ou seja, para o uso intencional de mecanismos de controle e autorregulação em favor dos objetivos de aprendizagem.

A formação do técnico em enfermagem demanda aprender a aprender conhecimentos teóricos e práticos, como também, a articulação entre demais profissionais da saúde e a assistência humanizada ao paciente (Serradilha, Duarte, \& Tonete, 2019). Nesse processo, a experiência de aprendizagem tem relação afetiva com experiências e crenças, sensação de saber e dificuldades, objetivos pessoais a serem alcançados e o desejo de promover a saúde na prestação de cuidados. Essa relação afetiva com a aprendizagem vai informar o processamento cognitivo, impactando na motivação e esforço a ser empregado na aprendizagem e conjuntamente, no controle que pode se dar por julgamentos de aprendizagem, de facilidade de aprendizagem, de compreensão, prévio e de confiança (Rosa et al., 2020; Efklides \& Metallidou, 2020).

A pesquisa da consciência metacognitiva apontou que a percepção do grupo para CM e RC foi razoável, com valores muito próximos nas duas categorias metacognitivas. Cabe salientar que o MAI foi aplicado de forma online, sem qualquer interferência dos pesquisadores, com a finalidade de obter um panorama geral do que os alunos pensam sobre si mesmos em relação ao próprio aprendizado. Deste resultado, podemos depreender que, na análise geral, há espaço para intervenções, no sentido ampliar e melhorar a consciência dos aprendizes em relação a sua própria aprendizagem. Além disso, faz-se necessário entender os motivos que levaram o fator depuração ter sido tão elevado em relação aos demais fatores. E, ainda, na análise individual, compreender os aspectos ou condições que podem ter influenciado cada resposta, diante de discrepâncias observadas entre CM e RC, acompanhadas pela grande quantidade de respostas “Às vezes”. Tais inferências, baseadas em Peixoto, Brandão e Santos (2007), nos levam a questionar: em que medida o conjunto de conceitos preexistentes dos alunos são adequados para lidar com sua própria aprendizagem?

Kuhn (2000) pontua que estudantes sofrem influência de crenças muitas vezes errôneas e não exercitam a autorreflexão. Nesse sentido, o percentual elevado para o fator depuração e respostas "Às vezes", pode estar relacionado a pouca atenção à escuta interior, sobre suas habilidades, dificuldades, conhecimento de estratégias de estudos e seus efeitos diante de situações práticas, ou seja, os resultados corresponderam a baixa ausculta, baixo conhecimento do self, e consequente pouco pensamento reflexivo sobre suas potencialidades e autorregulação de suas atividades educativas.

Além disso, como ressaltam Ertmer e Newby (1996), muitos estudantes não são adeptos da autoavaliação cognitiva. Dentre os fatores de RC o menor valor ocorreu para o fator Avaliação (63\%). Vale destacar, ainda, que o estudante que afirmou "sempre uso a mesma estratégia em todas as matérias, pois para mim é a melhor forma de aprender, por isso não mudo", apresentou $35 \%$ de conhecimento metacognitivo e $66 \%$ de regulação da cognitiva, indicando baixíssimo conhecimento de si e de estratégias, mas, em comparação, afirmou relativa capacidade para se autorregular e ajustar soluções diante dos erros. Diante disso, é pouco provável que um estudante com pouco conhecimento de suas potencialidades, de recursos e estratégias de aprendizagem, e que, portanto, avalie incompletamente seu processo de aprendizagem, tenha efetivas condições 
para identificar e corrigir seus erros de forma eficaz.

Em geral, por diversas razões, o aluno na escola reconhece a necessidade de aprender os conteúdos ensinados, mas um aspecto que se apresenta neste estudo, é o desconhecimento de seu papel nesse processo. O que significa dizer, o que se aprende sobre a aprendizagem, conhecimento de estratégias de aprendizagem, desenvolvimento da memória, exercícios de reflexão, resolução de problemas e resolução de conflitos. Foi exatamente isso, o conhecimento metacognitivo, que se buscou mensurar através das questões referentes aos três subprocessos ou fatores metacognitivos elencados por Schraw e Dennison (1994), conhecimentos declarativo, processual e condicional.

Contudo, convém destacar que o conhecimento metacognitivo pode ser impreciso, como alertam Efklides e Metallidou (2020). As autoras ressaltam que o conhecimento prévio fornece os padrões para julgar a precisão de uma resposta, assim, alunos com baixo desempenho muitas vezes não estão cientes de sua falta de conhecimento e/ou de suas habilidades. Se estudantes não percebem que desconhecem aspectos metacognitivos, é improvável que façam qualquer esforço para adquirir, escolher e aplicar adequadamente uma estratégia melhor, levando a ineficiência na utilização de estratégias de aprendizagem em decorrência de conhecimentos metacognitivos inadequados e de fraco monitoramento (Rosa et al., 2020; Santos, et al., 2021).

Além disso, Efklides e Metallidou (2020) relacionaram o baixo conhecimento prévio ou a falta de habilidade, com o excesso de confiança. A imprecisão de conhecimentos ou o excesso de confiança podem levar ao desvio de atenção a informações negativas ou a investir menos esforço do que o necessário em uma dada tarefa (Efklides \& Metallidou, 2020). De modo semelhante, tais questões podem ter influenciado nas respostas dadas pelos participantes que registraram percentual discrepante entre conhecimento metacognitivo e regulação cognitiva, demonstrando por vezes baixa percepção de si, de objetivos e de estratégias (CM), porém alta percepção sobre a gerência do ambiente (RC), planejamento de ações e uso de estratégias, avaliação de desempenho e correção de erros; ou percepção inversa com alto CM e baixo RC.

Outro aspecto a ser considerado, diz respeito às respostas automáticas ou inconscientes, que ocorrem sem que haja intervenção consciente ou decisão após reflexão (Efklides \& Metallidou, 2020). Neste caso, se o processamento cognitivo falhar, sem uma percepção consciente não saberemos como corrigir, pois, ocorre na forma de "piloto automático", como descreveu Rosa et al. (2020, p. 709). Esse tipo de ação automatizada, pode ter influenciado o aluno que mencionou sempre usar a mesma estratégia de aprendizagem, representando baixa reflexão sobre a experiência cognitiva e sobre estratégias e seus usos, como, quando e porquê usá-las.

A metacognição, que compreende a aquisição, desenvolvimento e acúmulo de conhecimento não é inata, é uma conquista evolutiva do ser humano. Ela se desenvolve, e pode ser ensinada (Kuhn, 2000; Jou \& Sperb, 2006; Efklides \& Metallidou, 2020; Xavier, Peixoto, \& Veiga, 2021). Deste modo, o desenvolvimento das capacidades metacognitivas repercute na capacidade de refletir sobre os próprios pensamentos, em sentimentos como confiança e em decisões acertadas, sendo aperfeiçoadas com o acúmulo do conhecimento transferido pela autorregulação da aprendizagem, como dispôs Lima Filho e Bruni (2015; Xavier, Peixoto, \& Veiga, 2021).

A autorregulação da aprendizagem está diretamente relacionada a metacognição e as funções de monitoramento e controle do processamento cognitivo. Segundo Efklides e Metallidou (2020), alunos autorregulados responsabilizam-se por sua aprendizagem. Estabelecem metas e são capazes de gerenciar o ambiente regulando: a cognição (monitorando e controlando a compreensão e o conhecimento); as emoções (afeto); a motivação (estímulo); e, o comportamento (desempenho).

Ertmer e Newby (1996) e Jou e Sperb (2006) defendem que um aprendiz experiente em conhecimento metacognitivo e habilidades metacognitivas reúne capacidade para saber o que é importante, como e porque utilizar recursos e conhecimentos adequados. Nesse sentido, Efklides e Metallidou (2020) e Xavier, Peixoto e Veiga (2021) apontam que competências metacognitivas podem ser ensinadas, visando dissipar a imprecisão, o baixo conhecimento prévio, o excesso de confiança, os 
bloqueios cognitivos e as ações automatizadas. Neste caso, o papel do professor pode ir além dos conteúdos, ao inserir em sua prática docente, o estímulo ao controle sobre o pensamento, o desempenho e a autorregulação, através de orientações para que alunos adotem, por exemplo, auto questões tais como: Como me sinto? O problema é fácil ou difícil? Como posso lidar com emoções negativas? Eu entendi o problema? O que é semelhante e o que é diferente? Qual é a estratégia que uso? Por que? A solução é razoável? Existe outra forma de solução? Quais? Como fazer?

Formulações como essas podem acionar a percepção metacognitiva e, conforme Midlej (2004, p 51), "torna-se uma verdadeira alavanca de formação interativa, produzindo-se, por meio de interiorização progressiva, condutas de desvelamento das experiências e das percepções, explicação, argumentação, aprimoramento do trabalho educacional e, espera-se, implementação de renovadas condutas". A conjunção entre expectativas de desempenho de aprendizagem, motivação pessoal, complexidade de disciplinas como Anatomia, Farmacologia e Projeto final, e ainda, atualmente, aprendizagens em ambientes virtuais, suscitam o aprimoramento do ensino regular e a construção da "cultura do pensar" (Veiga, Peixoto, \& Silva, 2021, p. 722).

De acordo com Flavell (1979), a metacognição ocorre de forma implícita, tornando-se difícil de avaliar, enfatiza Sales Júnior (2019). Entretanto, o MAI apresentou resultados confiáveis após a análise de confiabilidade e consistência interna dos indicadores, revelando-se uma excelente medida da metacognição (Schraw \& Dennison, 1994; Lima Filho, 2013; Lima Filho \& Bruni, 2015; Pinheiro, 2016).

Nesse estudo, o MAI apontou para a necessidade de se ampliar a consciência metacognitiva dos educandos, visto que apenas cinco participantes registraram resultados superiores a $80 \%$. Também, revelou que a maioria dos alunos do curso de técnico em Enfermagem estão dispostos a fazer aulas online para aprender a aprender, corroborando com o que explicam Veiga, Peixoto e Silva (2021, p. 737), "entender como se aprende melhor para que os conhecimentos adquiridos sejam realmente significativos para toda a vida do estudante".

Santos (2008) sinaliza que a aprendizagem deve contemplar a construção de destrezas cognitivas e conhecimentos, que podem ser interpretados como a apropriação de mecanismos de busca e seleção de informação, processos de análise e resolução de problemas, visando a autonomia progressiva no aprender do estudante, bem como, para o indivíduo como um todo, refletindo em sua vida.

Considerando o campo do ensino técnico em enfermagem, Serradilha, Duarte e Tonete (2019) propõem a adoção de metodologias ativas que possam promover a interação entre teoria e prática, a fim de envolver e significar a ação educativa, e que incentive a reflexão crítica da realidade e a proatividade do educando, futuro profissional da saúde.

Segundo Weber (2018), a metodologia ativa está ancorada na pedagogia crítica, que se contrapõe ao ensino tradicional, destacando o uso de situações-problema para o estímulo à aquisição de conhecimentos e habilidades. Morán (2015, p. 19) ressalta que "nas metodologias ativas de aprendizagem, o aprendizado se dá a partir de problemas e situações reais; os mesmos que os alunos vivenciarão depois na vida profissional, de forma antecipada, durante o curso".

Efklides e Metallidou (2020) suscitam que desenvolver atividades educativas a partir de intervenções alternativas, que envolvam habilidades metacognitivas e experiências subjetivas, afeto e motivação, podem aumentar a autoconsciência e facilitar a aprendizagem. Deste modo, na medida em que a educação em tempos pandêmicos mobilizou novas formas de ensino-aprendizagem, como o ensino híbrido, valorizar formas lúdicas de aprendizagem podem proporcionar um ambiente motivador para a aprendizagem e estímulo ao pensamento reflexivo (Tzohar-Rosen \& Kramarski, 2014).

\section{Considerações Finais}

Nesta pesquisa o Inventário de Consciência Metacognitiva foi realizado para delinear um panorama geral do que os alunos pensam sobre si e seu próprio aprendizado. O diagnóstico da percepção metacognitiva dos alunos do curso técnico em 
enfermagem, apontou que estes apresentaram um resultado razoável, tanto para o conhecimento de seu próprio conhecimento (conhecimento metacognitivo), quanto para a habilidade de monitoramento e controle de seu conhecimento (regulação da cognição).

Este resultado expressa que a percepção metacognitiva desses alunos ainda pode ser ampliada e melhorada, em relação ao desenvolvimento da consciência metacognitiva, em todos os fatores metacognitivos. Visto que, com melhor desenvolvimento metacognitivo, os alunos se tornam mais conscientes de seu próprio pensamento, do que conhecem e do que aprendem, com isso tendem a melhorar a performance cognitiva.

Cabe salientar que a percepção metacognitiva pode ser impactada pelo baixo conhecimento de si, baixo conhecimento prévio de conceitos e estratégias, informações imprecisas, excesso de confiança, bloqueios cognitivos e ações automatizadas. Neste aspecto, o professor poderia adotar metodologias ativas de aprendizagem, atuar inserindo estratégias de estímulo à cultura do pensar para promover a reflexão crítica e a autorregulação, e/ou novas estratégias de ensino-aprendizagem.

Além disso, a formação do técnico em enfermagem deve compreender competências técnicas, mas também habilidades e atitudes, como capacidade de reflexão, proatividade e autonomia, para um cuidado integral e humano. Deste modo, suscita a busca por novos caminhos no ensino-aprendizagem, que permita promover o pensamento crítico e a autoconsciência em relação à própria aprendizagem.

Nessa perspectiva, trabalhos futuros podem explorar intervenções pedagógicas que incluam interações entre metacognição e afeto, por exemplo, através de trabalhos que envolvam metodologias ativas como aprendizagem baseada em problemas, gamificação e simulação realística, visando o repensar dos próprios conhecimentos, tomada de consciência, autoconfiança, tomada de decisão e motivação para aprender. E, ainda, estudos que estimulem o conhecimento da metacognição e uso de estratégias metacognitivas na prática docente.

\section{Referências}

Babbie, E. (2003). Métodos de Pesquisas de Survey. Tradução Guilherme Cezarino. Editora UFMG.

Bacich, L, Tanzi Neto, A., \& Trevisani, F. M. (2015). Ensino híbrido: personalização e tecnologia na educação. Organizadores, Lilian Bacich, Adolfo Tanzi Neto, Fernando de Mello Trevisani. e-PUB, Penso.

Brasil. (2016). Resolução CNS no 510 de 7 de abril de 2016. Ministério da Saúde. Conselho Nacional de Saúde. https://bit.ly/33i4RES

Brasil. (2020). Medida Provisória $n^{\circ} 934$, de $1^{\circ}$ de abril de 2020. Estabelece normas excepcionais sobre o ano letivo da educação básica e do ensino superior decorrentes das medidas para enfrentamento da situação de emergência de saúde pública de que trata a Lei nº 13.979 , de 6 de fevereiro de 2020 . Brasília, 2020 . https://bit.ly/3ksoQqu.

Efklides, A., \& Metallidou, P. (2020) Applying Metacognition and Self-Regulated Learning in the Classroom. Oxford Education. https://doi.org/10.1093/acrefore/9780190264093.013.961

Ertmer, P. A., \& Newby, T. J. (1996). The expert learner: Strategic, self-regulated, and reflective. Instructional Science, 24 , 1-24. https://doi.org/10.1007/BF00156001

Flavell, J. H, Miller, H. P., \& Miller, S. A. (1999). Desenvolvimento cognitivo. Trad. Claudia Dornelles, Artmed.

Flavell, J. H. (1979). Metacognition and cognitive monitoring: a new area of cognitive-developmental inquiry. American Psychologist, 34, 906-911.

Hodges, C., Moore, S., Lockee, B., Trust, T., \& Bond, A. (2020). The Difference Between Emergency Remote Teaching and Online Learning. Educause Review. https://bit.ly/3fgvpZM

Jou, G. I. de, \& Sperb, T. M. (2006). A metacognição como estratégia reguladora da aprendizagem. Psicol. Reflex. Crit. [online], 19(2), 177-185. https://cutt.ly/MzhB9HD

Kuhn, D. (2000). Metacognitive Development. Current Directions In Psychological Science, EUA, 9(5), 178-181. https://doi.org/10.1111/1467-8721.00088

Lima Filho, R. N. (2013). Empreendendo sobre o empreender e pensando sobre o pensar: um estudo sobre características empreendedoras e metacognição. Tese de doutorado, Curso de Administração, Escola de Administração, Universidade Federal da Bahia, Salvador, BA, Brasil. https://cutt.ly/azh84Up.

Lima Filho, R. N., \& Bruni, A. L. (2015). Metacognitive Awareness Inventory: tradução e validação a partir de uma análise fatorial confirmatória. Psicologia: Ciência e Profissão, 35(4), 1275-1293. https://doi.org/10.1590/1982-3703002292013 
Marin, M. J. S., Dias, M. U. S. S., Tonhom, S. F. da R., Michelone, A. P. C., \& Bernardo, M. do C. M. (2014). Estudantes de curso técnico em enfermagem e sua motivação para o trabalho em enfermagem. Rev. Eletr. Enf. [Internet]. 16(2):401-7. http://dx.doi.org/10.5216/ree.v16i2.20827

Midlej, J. (2004). Os diários reflexivos e os processos metacognitivos na práxis educacional. Aprender - Caderno de Filosofia e Psicologia da Educação, Ano II(3), 49-61. https://bit.ly/2XGEkxU

Morán, J. (2015). Mudando a educação com metodologias ativas. Coleção Mídias Contemporâneas. Convergências Midiáticas, Educação e Cidadania: aproximações jovens, Vol. II, Carlos Alberto de Souza e Ofelia Elisa Torres Morales (orgs.), PG: Foca Foto-PROEX/UEPG, pp. 15-33. https://cutt.ly/Kzh1w7L

Peixoto, M. A. P., Brandão M. A. G., \& Tavares, B. F. (2021). Construção de definições operacionais em Metacognição. SciELO Preprints. https://doi.org/10.1590/SciELOPreprints. 2331

Peixoto, M. A., Brandão, M. A., \& Santos, G. dos. (2007). Metacognição e tecnologia educacional simbólica. Rev. Educ. Med., 31(1), 67-80. https://doi.org/10.1590/S0100-55022007000100010

Pinheiro, R. P. Q. (2016). “APRENDE-TE”! O papel do não cognitivo na aprendizagem. Dissertação de Mestrado, Curso de Psicologia Clínica, ISPA Instituto Universitário, https://cutt.ly/Mzh0xQr

Pintrich, P. R. (2002). The Role of Metacognitive Knowledge in Learning, Teaching, and Assessing. Theory Into Practice, Vol. 41, No. 4, Revising Bloom's Taxonomy, pp. 219-225, Published by: Taylor \& Francis, Ltd. https://cutt.ly/Fzh0Hhs

Rosa, C. T. W. da, Corrêa, N. N. G., Passos, M. M., \& Arruda, S. de M. (2020). Metacognição e seus 50 anos: uma breve história da evolução do conceito. Revista Educar Mais, 4(3), 703-721. https://doi.org/10.15536/reducarmais.4.2020.2063

Sales Júnior, V. B. de. (2019). Identificando a necessidade do conhecimento metacognitivo em ingressantes de instituições de ensino superior através do inventário de consciência metacognitiva. IX Encontro de Pesquisa em Educação de Alagoas (EPEAL), 1, 1-8. https://cutt.ly/Gzh2cxz.

Santos, J. da C. M. dos. (2008). Orientações Motivacionais e Estratégias de Aprendizagem no Ensino Superior: Contributos para a compreensão da assiduidade às aulas dos estudantes de Enfermagem. Dissertação de Mestrado, Curso de Educação e Desenvolvimento Social. Universidade de Coimbra, Coimbra, https://cutt.ly/8zh2KEL

Santos, L. R. dos, Albuquerque, C. F. Da S., Veiga, L. L. de A. da, \& Peixoto, M. A. P. (2021) O Ensino Remoto Emergencial na Perspectiva da Metacognição: Análise da Percepção de Alunos de um Curso Técnico em Enfermagem. EaD Em Foco, 11(2). https://cutt.ly/LbESxl1

Schraw, G., \& Dennison, R. S. (1994). Assessing metacognitive awareness. Contemporary Educational Psychology, 19, (4), 460-475. https://doi.org/10.1006/ceps.1994.1033

Serradilha, A. F. Z., Duarte M. T. C., \& Tonete, V. L. P. (2019). Health promotion by nursing technicians from the nurses' perspective. Revista Brasileira de Enfermagem, 72(4), 979-987. https://doi.org/10.1590/0034-7167-2018-0552

Silva, M. R., Chini, L. T., Silva, T. O., Martinez, M. R., \& Sanches, R. S. (2018). Competências dos profissionais técnicos de enfermagem: o que esperam os enfermeiros gestores? Enferm. Foco, Brasil, 4(9), 66-72. https://doi.org/10.21675/2357-707X.2018.v9.n4.1335

Tzohar-Rosen, M., \& Kramarski, B. (2014). Metacognition, motivation and emotions: Contribution of self-regulated learning to solving mathematical problems. Global Education Review. 1 (4), p. 76-95. https://cutt.ly/Zzh3QNd

Veiga, L. L. de A. da, Peixoto, M. A. P., \& Silva, C. X. (2021). Diários de campo, metacognição e aprendizagem: o que podemos observar em alunos de uma disciplina do tipo "aprender a aprender". Debates em Educação, 13(31), 719-743. https://doi.org/10.28998/2175-6600.2021v13n31p719-743

Weber, L. C. (2018). Metodologias ativas no processo de ensino da enfermagem: revisão integrativa. Dissertação de mestrado em Ensino, Ciência, Sociedade e Ensino, Universidade do Vale do Taquari - Univates, https://cutt.ly/Yzh39EW

Wermelinger, M. C. de M. W. (2007). Educação profissional: o técnico da saúde (enfermagem) em evidência. Tese de doutorado, Curso de Escola Nacional de Saúde Pública Sergio Arouca, Fiocruz, https://cutt.ly/szh8EKc

Xavier, C. S., Peixoto, M. A. P., \& Veiga, L. L. de A. da. (2021) Embedded metacognitive prompts based on the nature of science: Potentialities, limitations, conditions and possibilities. Research, Society and Development, 10(7), e43010716829. http://dx.doi.org/10.33448/rsd-v10i7.16829 